
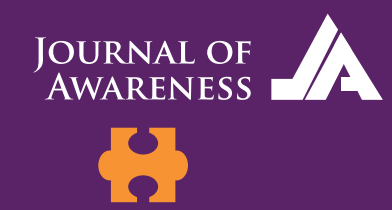

\title{
Associations between biometric characteristics and occupational safety and health
}

\section{Lourdes Ruiz Salvador ${ }^{1}$ (D) Tibor Kovacs² ${ }^{2}$ (D) Richard Perez}

1 Ph.D. Obuda University, Banki Donat Faculty, Doctoral School on Safety and Security Sciences, Budapest, Hungary, e-mail: lourdes.ruiz@uni-obuda.hu 2 Prof. Dr. Obuda University, Banki Donat Faculty, Budapest, Hungary, email: ovacs.tibor@uni-obuda.hu

3 MD, Universidad Tecnica de Cotopaxi, Department of Occupational Health, Latacunga, Ecuador, email: richard.perez@utc.edu.ec

\begin{abstract}
Any job comes with different occupational hazards. Office-bounded positions had become pervasive over the past years. Moreover, the pandemic crisis experienced in 2020 shifted these jobs to a home office, generating unexpected hazards. The case study presented below collected primary data from 409 university employees through biometric screenings and analyzed it using descriptive and inferential statistics. Chi-square tests established statistically significant associations between type of occupation and characteristics such as gender, body mass index, blood and urine sample laboratory results, and age. Logistic regression determined two significant factors that contribute to occupational diagnosis (gender and physical exam results). In addition, the study identified clinical problems and pathologies related to mental work. These results were pivotal for identifying specific work hazards such as obesity, musculoskeletal disorders, eye problems, and metabolic diseases and how they affect health and safety in the workplace.
\end{abstract}

Keywords: Occupational, Safety, Health, Hazards, biometric characteristics 


\section{INTRODUCTION}

According to the International Labour Organization (ILO), around 2.3 million people worldwide suffer occupational accidents and diseases every year, with over 6000 work-related deaths per day(ILO, 2021). These accidents bear a significant impact on society and the economy. Specifically, in the European Union, the cost of workplace incidents is estimated at $€ 479$ billion per year, while in the United States accounts for \$ 161.5 billion(NSC, 2019). Consequently, worldwide occupational injuries and illnesses represent a loss of $3.9 \%$ of GDP, equivalent to a total cost of $€ 2680$ billion(EU-OSHA, 2017). Hence, occupational safety and health $(\mathrm{OSH})$ practices are essential for keeping sustainable and productive businesses.

OSH's primary goal is to prevent risks and occupational hazards to safeguard the employees' health(Alli, 2008). An occupational hazard is any condition in the work environment that can cause a risk of illness or accident to the employee. They are divided into six categories: safety, biological, chemical, physical, ergonomic, and organizational(Shaw, 2021). Any profession comes with specific hazards. For example, office work has become prevalent nowadays. Furthermore, due to the pandemic, the office landscape shifted to households bringing unexpected challenges in safety and health. Mental work consists of tasks that principally require thinking, reasoning, and planning in contrast to physical work mainly executed by the muscles and bones in the body(Fisher et al., 2014).

Biometric screenings constitute a powerful tool to enhance safety and health in the workplace. These screenings measure biometric characteristics such as height, weight, blood pressure, cholesterol, and more to recognize worker's health risks and evaluate their health status throughout time(CDC, 2018).

The following work presents a case study that acquired primary data via biometric screenings from 409 university employees in Ecuador. It identified mental work-related hazards to create awareness of the risks of these types of jobs and formulate better practices to alleviate these issues. Furthermore, inferential statistical tests such as chi-square and logistic regression showed significant relations between biometric characteristics and occupational diagnosis. These results demonstrate how biometric characteristics affect workforces' health and their ability to perform the tas- ks they are hired to perform safely.

\section{METHODOLOGY}

Biometric characteristics such as height, weight, gender, type of occupation, blood and urine samples were collected from 409 university employees as part of the annual biometric screening program. Additionally, the occupational doctor performed a physical examination and an occupational diagnosis establishing if a worker is fit to work, fit with limitations, or unfit to work. This diagnosis is a clinical evaluation that considers the employee's biometric screening results with the working conditions, environment, and specific job tasks to guarantee that the workers do not constitute a hazard for themselves, colleagues, and the compan$y(C C O H S, 2016)$.

Descriptive statistics were performed to characterize the population and find common affections among the workforce. Inferential statistic tests were executed using IBM SPSS software. Chi-Square tests for independence were done to establish statistically significant associations between biometric characteristics and occupational diagnosis. In addition, logistic regression was executed to determine what factors influence occupational diagnosis.

Chi-Square tests help identify two categorical variables' dependency, but they cannot establish the nature, direction, or strength of this relationship. Consequently, other methods, such as logistic regression, are needed to understand how these biometric traits influence occupational diagnosis(Gilbert and Prion, 2016).

Logistic regression is popular among health sciences. In comparison to Chi-square tests that analyze two categorical variables simultaneously. It can analyze a mix of variables (continuous, categorical) and determine their effect on a discrete outcome. Logistic regression was performed to assess the factors that influence occupational diagnosis. The biometric characteristics acquired during the biometric screening event were used in the model. The model comprised of eleven predictors: nine categorical variables (gender, age, alcohol, tobacco consumption, laboratory results, physical exam results, pathologies, clinical problems, and type of occupation) and two continuous variables (height and weight). 


\section{RESULTS}

Table 1 presents several participants' biometric characteristics. Regarding gender, males constitute a larger percentage of employees compared with females. Participants, aged 15 to 41 years, represent a higher percentage than their colleagues over 41 years old. A relatively small number of participants perform physical work than mental work. Concerning the measured biometric traits such as height and weight, the mean height is within Ecuadorians' normal ranges. However, there are extreme values to pay attention to obesity and diseases; this is reflected in the high standard deviation values, representing variation in the mean weight group. The occupational analysis revealed that nearly $84 \%$ of the employees are apt to work in their current job position, more than $16 \%$ are apt to work but with specific restrictions. Consequently, there are no cases of employees that are classified as unfit to work.

Table 2 summarizes the main findings obtained in the biometric screening event. The overall BMI classification shows a considerable percentage $(60 \%)$ of overweight or obese employees, and nearly $40 \%$ have a normal BMI. Furthermore, the occupational doctor diagnosed clinical problems and pathologies by analyzing the laboratory and physical exam results. Clinical problems such as polycythemia, sedentarism, hypercholesterolemia, and hypertriglyceridemia are prevalent among university workers. As for the pathologies, metabolic, hematologic are predominant. Ophthalmologic and musculoskeletal pathologies comprise significant percentages $(38.6 \%, 19 \%)$, respectively.

Table 1. Population Demographics

\begin{tabular}{llr}
\hline Biometric Characteristics & Value \\
\hline Gender & Male \% & 60.6 \\
\cline { 2 - 3 } & Female\% & 39.4 \\
\hline \multirow{2}{*}{ Age Range } & $15-41$ years \% & 58.7 \\
\cline { 2 - 3 } & $>$ 41 years \% & 41.3 \\
\hline Type of Occupation & Physical Work \% & 11.7 \\
\cline { 2 - 3 } & Mental Work \% & 88.3 \\
\hline Height (meter) & Mean Height (Standard Deviation) & $1.63(0.08)$ \\
\hline Weight (kilogram) & Mean Weight (Standard Deviation) & $71.45(12.98)$ \\
\hline Occupational Diagnosis & Fit \% & 83.4 \\
\cline { 2 - 3 } & Fit with limitations \% & 16.6 \\
\hline
\end{tabular}

Table 2. Biometric Screening Results

\begin{tabular}{llr}
\hline Results & & Normal \\
\hline BMI Classification & Overweight & 38.9 \\
\cline { 2 - 3 } & Obesity & 44 \\
\cline { 2 - 3 } & Morbid Obesity & 16.4 \\
\hline Clinical Problems & Sedentarism & 0.7 \\
\cline { 2 - 3 } & Polycythemia & 57 \\
\cline { 2 - 3 } & Hypercholesterolemia & 56.5 \\
\cline { 2 - 3 } & Hypertriglyceridemia & 46.2 \\
\cline { 2 - 3 } & Hyperuricemia & 36.7 \\
\cline { 2 - 3 } & Fatty liver & 10.5 \\
\cline { 2 - 3 } & Hypertension & 9.1 \\
\hline Pathologies & Metabolic & 7.6 \\
\cline { 2 - 3 } & Hematologic & 78 \\
\cline { 2 - 3 } & Ophthalmological & 38.3 \\
\cline { 2 - 3 } & Musculoskeletal & 19.1 \\
\hline Problems in the Physical Examination & Eyes & 38.9 \\
\cline { 2 - 3 } & &
\end{tabular}


Figure 1. Statistical association between biometric characteristics

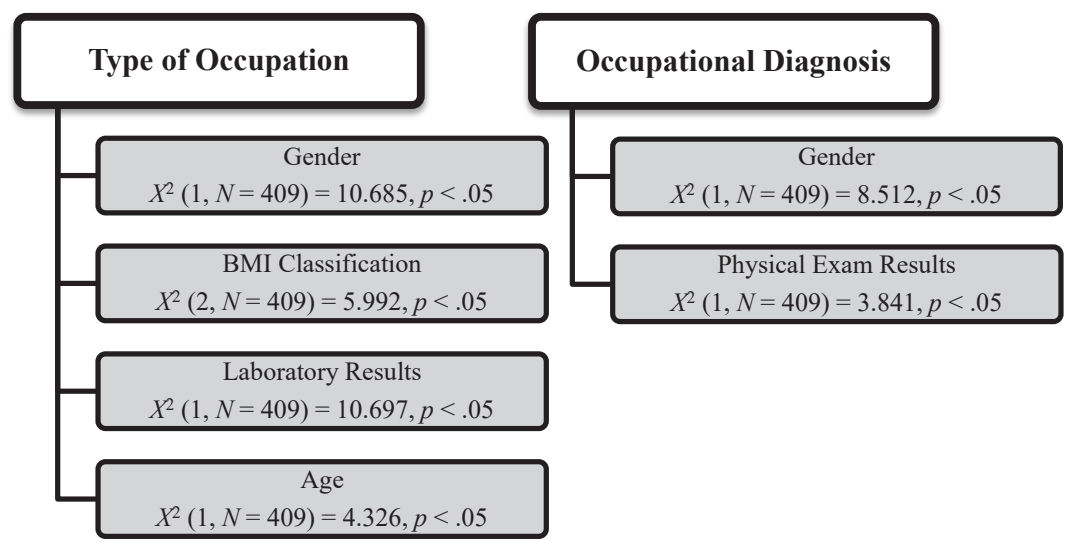

\subsection{Chi-Square Tests}

The Chi-Square test constitutes a first approach to determine the association between the biometric characteristics analyzed. Statistically significant relationships at $\mathrm{p}<0.05$ were established. Figure 1 enumerates the relations identified between the type of occupation, occupational diagnosis, and several biometric characteristics.

As Figure 1 portrays, the type of occupation showed a robust association with four biometric traits: gender, age, BMI classification, and the results obtained via blood and urine tests. Likewise, occupational diagnosis is statistically related to gender and physical exam results.

\subsection{Logistic Regression}

The model was statistically significant $(\chi 2=46.694, \mathrm{df}=$ $11, \mathrm{p}<0.001, \mathrm{~N}=409$ ), which means it could differentiate between the employees diagnosed as fit to work and fit with limitations to work. As indicated in Table 3 , the two major factors influencing occupational diagnosis are gender and the results obtained via physical exams. Physical exam results are a sturdy predictor of the model with an odds ratio value of approximately 4 , suggesting that workers who present physical exam problems are four times more likely to be diagnosed as fit with limitations to work. Concerning gender, the odds ratio value was 0.295 , which indicates that a person's odds to be diagnosed as fit with limitations to work decreases by a factor of 0.295 for a male emplo- yee; all other factors in the model controlled.

\section{DISCUSSION}

This study delivered valuable information regarding the university workforce's health. Biometric screening results pointed out commonalities in abnormal laboratory results, physical exams, clinical problems, and pathologies. These results and the occupational diagnosis were used to perform inferential statistical analyses such as chi-square tests and logistic regression.

Chi-square test results indicated a significant association between the type of occupation (mental, physical work) and the following biometric characteristics: gender, BMI classification, laboratory results, and age. Also, occupational diagnosis (fit to work, fit with limitations) is related to the biometric characteristics of gender and physical exam results.

In addition, logistic regression identified two main factors that impact the occupational diagnosis: gender and physical examination. Therefore, in this specific case having problems in the physical exam and gender can influence the ability to perform a job.

Most employees perform mental work (88\%), such as office assistant, professor, administrative work, and others. This type of occupation is linked to specific hazards due to the nature of the work. In this particular case study, the following mental-related work hazards were identified and presented in Figure 2.

Table 3. Associated factors to Occupational Diagnosi

\begin{tabular}{|l|r|l|l|}
\hline Factors & \multicolumn{1}{|l|}{ B } & \multicolumn{1}{|c|}{ Adjusted Odds Ratio (95\% CI) } & $\boldsymbol{P}$ - value \\
\hline Gender & -1.219 & $0.295(0.149,0.587)$ & $<0.001$ \\
\hline Physical Exam Results & 1.356 & $3.879(2.051,7.336)$ & $<0.001$ \\
\hline
\end{tabular}


Figure 2. Identified mental work hazards

\begin{tabular}{|c|}
\hline BMI \\
Classification \\
\hline \\
• Pre obesity \\
- Overweight \\
• Obesity \\
• Morbid \\
Obesity \\
\hline
\end{tabular}

\begin{tabular}{|c|}
\hline $\begin{array}{c}\text { Abnormal } \\
\text { Laboratory } \\
\text { Results }\end{array}$ \\
\hline \\
$\bullet$ High \\
cholesterol \\
$\bullet$ High \\
triglycerides \\
$\bullet$ High \\
hematocrit \\
\hline
\end{tabular}
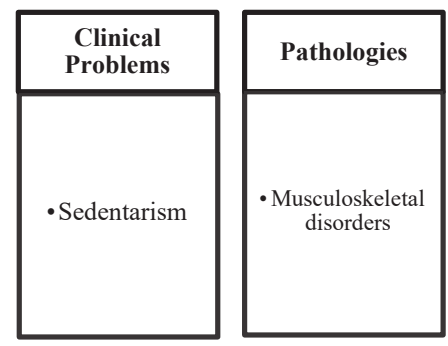

These results also prove how a particular work affects the employees' health, which should be an apparent reason for changing the work environment that can impact workplace safety and health.

The first step in a risk assessment is hazard identification(Risk Assessment: OSH Answers, no date). Thanks to biometric screenings, bounded employee hazards can be easily spotted. Moreover, the analysis of the results can provide a tailored hazards profile of the specific job position. This identification creates awareness and can aid in hazard prioritization. Thus, hazard identification serves as the grassroots for directing safety and health policies in the university. Additionally, it researches the causes, provides evidence of the hazards related to desk-bound professions, and identifies specific actions and policies to treat and manage these conditions.

As a consequence of the pandemic, mental work drifted to households, posing different challenges and changing the occupational safety and health dynamics. Teleworking has several advantages, such as schedule flexibility, lack of commuting, and the possibility of working from any location. However, it can provoke several problems such as more distractions, less productivity, loneliness, and unsafe work environments in terms of ergonomics, extended screen time, and difficulty disconnecting from work tasks (overworking) (Fogarty et al., 2020; Routley, 2020). Biometric screening results can help identify home office hazards and workers' actual health and safety consequences due to the Covid-19 outbreak.

\section{CONCLUSIONS}

Nowadays, biometric screenings are utterly needed to assess the remote and onsite workers' wellbeing and analyze how the pandemic affects them. Screenings can detect long-term effects in an infected employee case or identify specific health conditions in workers more vulnerable to the virus to instate preventive me- asures and plan better treatment options. Moreover, it is vital to pay extra attention to new high-risk professions such as essential service employees, healthcare, the food industry, and public transportation. These covid-19 risk jobs take a disproportionate toll on racial and ethnic minorities that require occupational safety protection. $\mathrm{OSH}$ initiatives and policies, including safety procedures during biometric screening events, must be generated and adapted to the new workplace reality to supply safe and healthy workspaces.

The impact of sedentary jobs on workers' health is catching the public's attention. Health policies are trying to shape the work environment, but they do not provide enough incentives to the corporations. Biometric screenings can be the initial point for improving health and giving the employer motivation by offering data that can impact safety that, in the long term, reduces costs and improves the workforce morale and the company image. In addition, it opens up the opportunity to discuss until what point the employer is responsible for the worker's health, lifestyle choices, and habits.

\section{REFERENCES}

ALLI, B. O. (2008) Fundamental principles of occupational health and safety, Vasa. doi: 10.1017/CBO9781107415324.004.

CCOHS (2016) Fit to Work: OSH Answers, Canadian Centre for Occupational Health and Safety. Available at: https://www.ccohs.ca/oshanswers/psychosocial/fit_to_work.html (Accessed: 11 November 2019).

CDC (2018) Workplace Health Glossary, Center for Disease Contral and Prevention: Workplace Health Promotion. Available at: https://www.cdc. gov/workplacehealthpromotion/tools-resources/glossary/glossary.html.

EU-OSHA (2017) Work-related accidents and injuries cost EU $€ 476$ billion a year according to new global estimates [1] Work-related accidents and injuries cost EU $€ 476$ billion a year according to new global estimates. Singapore. Available at: https://osha.europa.eu/en/about-eu-osha/press-room/ eu-osha-presents-new-figures- (Accessed: 19 July 2021).

FISHER, G. G. et al. (2014) 'Mental work demands, retirement, and longitudinal trajectories of cognitive functioning', Journal of Occupational Health 
Psychology. Educational Publishing Foundation, 19(2), pp. 231-242. doi: 10.1037/a0035724.

FOGARTY, P. et al. (2020) Coronavirus: How the world of work may change forever?, BBC: Worklife. Available at: https://www.bbc.com/worklife/ article/20201109-coronavirus-how-cities-travel-and-family-life-will-change (Accessed: 13 January 2021).

GILBERT, G. E. \& PRION, S. (2016) 'Making Sense of Methods and Measurement: The Chi-Square Test', Clinical Simulation in Nursing. International Nursing Association for Clinical Simulation and Learning, 12(5), pp. 145-146. doi: 10.1016/j.ecns.2015.12.013.

ILO (2021) World Statistic: The enormous burden of poor working conditions, International Labour Organization. Available at: https://www.ilo.org/ moscow/areas-of-work/occupational-safety-and-health/WCMS_249278 lang--en/index.htm (Accessed: 19 July 2021)

NSC (2019) Work Injury Costs - Injury Facts, National Safety Council. Available at: https://injuryfacts.nsc.org/work/costs/work-injury-costs/ (Accessed: 19 July 2021).

Risk Assessment : OSH Answers (no date). Available at: https://www.ccohs. ca/oshanswers/hsprograms/risk_assessment.html (Accessed: 9 December 2019).

ROUTLEY, N. (2020) Coronavirus: Is working from home working? , World Economic Forum. Available at: https://www.weforum.org/agenda/2020/06/ coronavirus-covid19-remote-working-office-employees-employers (Accessed: 13 January 2021)

SHAW, G. (2021) Occupational Hazards: An Overview, WebMD. Available at: https://www.webmd.com/a-to-z-guides/occupational-hazards (Accessed: 20 July 2021) 